\title{
Designing displaced lunar orbits using low-thrust propulsion
}

\author{
Jules Simo* and Colin R. McInnes ${ }^{\dagger}$ \\ University of Strathclyde, Glasgow, G1 1XJ, United Kingdom
}

\section{Introduction}

$\mathbf{T}$ HE use of solar electric propulsion (SEP) technology is now a realistic option for designing trajectories for interplanetary missions, while solar sail technology is currently under development. This paper covers the results of a study on displaced periodic orbits in the Earth-Moon system in which the third body uses a hybrid solar sail. The hybrid sail model is composed of two low thrust propulsion systems, namely a solar sail and solar electric propulsion.

A solar sail is propelled by reflecting solar photons; transforming the momentum of the photons into a propulsive force. Solar sail technology appears to be a promising form of advanced spacecraft propulsion which can enable exciting new space-science mission concepts such as solar system exploration and deep space observation. This form of propulsion can in principle provide energy changes greater than are possible with either ion or chemical propellants. Solar sails can also be utilised to maintain highly non-Keplerian orbits, such as closed orbits displaced high above the ecliptic plane (see McInnes, ${ }^{1}$ Waters and McInnes, ${ }^{2}$ Simo and $\mathrm{McInnes}^{3}$ ). Solar sails are especially suited for such non-Keplerian orbits, since they can apply a propulsive force continuously over long periods. In such trajectories, a sail can be used as a communication satellite for high latitudes. For example, the orbital plane of the sail can be displaced above the orbital plane of the Earth, so that the sail can stay fixed above the Earth at some distance, if the orbital periods are equal. McInnes ${ }^{4}$ investigated a new family of displaced solar sail orbits near the Earth-Moon libration points. Displaced orbits have more recently been developed by Ozimek et al. ${ }^{5}$ using collocation methods. In Baoyin and McInnes ${ }^{6,7,8}$ and McInnes ${ }^{4,9}$, the authors describe new orbits which are associated with artificial Lagrange points in the Earth-Sun system. These artificial equilibria have potential applications for future space physics and Earth observation missions. In McInnes and Simmons ${ }^{10}$, the authors investigate large new families of solar sail orbits, such as Sun-centered halo-type trajectories, with the sail executing a circular orbit of a chosen period above the ecliptic plane.

The idea of combining a solar sail with an auxiliary SEP system to obtain a hybrid sail system is important due to the challenges of performing complex missions (see Leipold and Götz, ${ }^{11}$ Mengali and Quarta, ${ }^{12}$ Dachwald, ${ }^{13}$ Baig and McInnes ${ }^{14}$ ). The solar electric propulsion system possesses high specific impulse $\left(I_{s p} \approx 3000 \mathrm{sec}\right)$. SEP consumes propellant and decreases the mass of the spacecraft, whereas the solar sail does not consume any propellant. This form of propulsion is useful for some high energy missions, but unlike solar sails, they have a finite $\Delta V$ capability, which makes them unsuitable for missions where a non-Keplerian orbit has to be maintained over indefinite periods of time.

Orbits around the collinear libration points of the Earth-Moon system are of great interest because their unique positions are advantageous for several important applications in space mission design (see e.g. Szebehely ${ }^{15}$, Farquhar, ${ }^{16}$ Roy ${ }^{17}$ Vonbun, ${ }^{18}$ Thurman et al., ${ }^{19}$ Gómez et al., ${ }^{20,21}$ Breakwell and Brown ${ }^{22}$, Richardson ${ }^{23}$, Howell ${ }^{24,25}$ ). Such orbits cannot be maintained without active control due to their instability (see Breakwell and Brown ${ }^{22}$, Richardson ${ }^{23}$, Howell ${ }^{24,25}$ ). If the orbit maintains visibility from Earth, a spacecraft on it (near the $L_{2}$ point) can be used to provide communications between the equatorial regions of the Earth and the lunar poles. Moreover, if another communications satellite is located at the $L_{1}$ point,

\footnotetext{
* Research Fellow, Advanced Space Concepts Laboratory, Department of Mechanical Engineering, jules.simo@strath.ac.uk.

†Professor, Advanced Space Concepts Laboratory, Department of Mechanical Engineering, colin.mcinnes@strath.ac.uk, Member AIAA.
} 
there could be continuous communications coverage between the equatorial region of the Earth and the entire lunar surface (see Farquhar ${ }^{26}$, Farquhar and $\mathrm{Kamel}^{27}$ ).

This paper investigates displaced periodic orbits at linear order in the circular restricted Earth-Moon system, where the third massless body utilizes a hybrid of solar sail and a solar electric propulsion system. In particular, periodic orbits in the vicinity of the Lagrange points in the Earth-Moon system will be explored along with their applications. Firstly we describe the dynamic model of the hybrid sail. The first-order approximation is derived for the linearized equations of motion. Then, a feedback linearization control scheme (see Slotine and $\mathrm{Li}^{28}$ ) is proposed and implemented. The main idea of this approach is to cancel the nonlinearities and to impose desired linear dynamics satisfied by the solar sail. We then select the SEP control, which takes into consideration the nonlinearity cancellation and the stabilizing linear control. When the control is applied to the nonlinear system, asymtotic stability is achieved. This provides the key advantage that the displacement distance of the hybrid sail is then constant. A constant displacement distance of $1750 \mathrm{~km}$ has been considered for the simulations. The displaced orbits found by Ozimek et al. ${ }^{5}$ show large excursions in displacement distance. In practice, a constant displacement distance may lead to easier tracking from the lunar surface for communications applications. Finally, we evaluate the performance of the hybrid sail approach.

\section{System Model}

In this work $m_{1}$ represents the larger primary (Earth), $m_{2}$ the smaller primary (Moon) and we will be concerned with the motion of a hybrid sail that has negligible mass. It is always assumed that the two more massive bodies are moving in circular orbits with constant angular velocity $\omega$ about their common center of mass, and the mass of the third body is too small to affect the motion of the two more massive bodies. The unit mass is taken to be the total mass of the system $\left(m_{1}+m_{2}\right)$ and the unit of length is chosen to be the constant separation $R^{\star}$ between $m_{1}$ and $m_{2}$. The time unit is defined such that $m_{2}$ orbits around $m_{1}$ in time $2 \pi$. Under these considerations the masses of the primaries in the normalized system of units are $m_{1}=1-\mu$ and $m_{2}=\mu$, with $\mu=m_{2} /\left(m_{1}+m_{2}\right)$ (see Figure 1 (a)). Thus, in the EarthMoon system, the nondimensional unit acceleration is $a_{r e f}=\omega^{2} R^{\star}=2.7307 \mathrm{~mm} / \mathrm{s}^{2}$ where the Earth-Moon distance $R^{\star}=384400 \mathrm{~km}$. The dashed line in Figure 1 (a)) is a line parallel to the Sun-line direction.

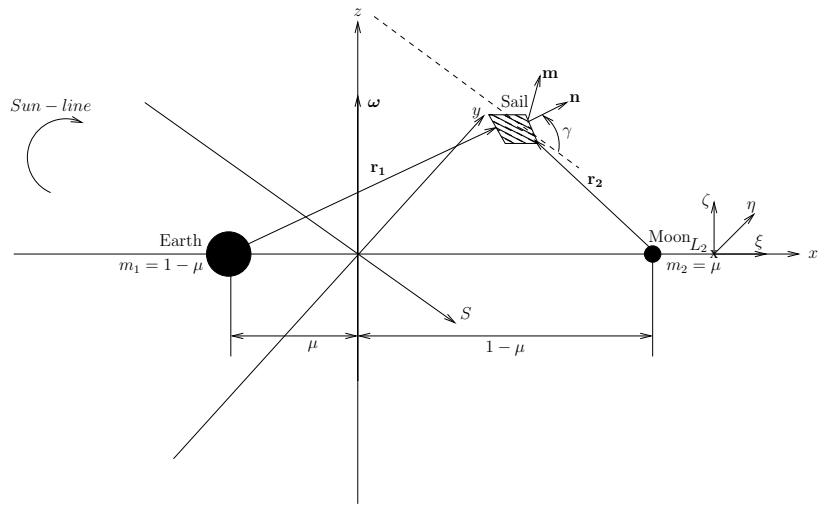

(a)

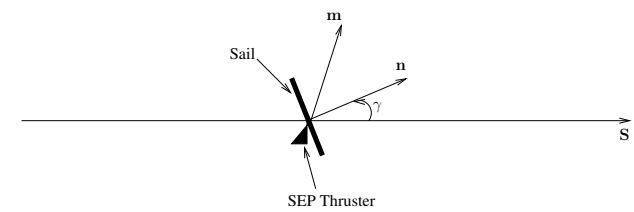

(b)

Figure 1. (a) Schematic geometry of the Hybrid Sail in the Earth-Moon circular restricted three-body problem; (b) Angle $\gamma$ between the Hybrid Sail surface normal $n$ and the Sun-line direction $S$, and SEP thrust vector direction $m$.

\section{II.A. Equations of Motions}

The nondimensional equation of a motion of a hybrid sail ${ }^{14}$ in the rotating frame of reference is described by

$$
\frac{d^{2} \boldsymbol{r}}{d t^{2}}+2 \boldsymbol{\omega} \times \frac{d \boldsymbol{r}}{d t}+\nabla U(\boldsymbol{r})=\boldsymbol{a}_{S}+\boldsymbol{a}_{S E P}
$$

where $\boldsymbol{\omega}=\omega \hat{\boldsymbol{z}}(\hat{\boldsymbol{z}}$ is a unit vector pointing in the direction $\boldsymbol{z}$ ) is the angular velocity vector of the rotating frame and $r$ is the position vector of the hybrid sail relative to the center of mass of the two primaries. We 
will not consider the small annual changes in the inclination of the Sun-line with respect to the plane of the system. The three-body gravitational potential $U(\boldsymbol{r})$, the solar radiation pressure acceleration $\boldsymbol{a}_{S}$ and the nondimensional acceleration due to the SEP thruster $\boldsymbol{a}_{S E P}$ are defined by

$$
\begin{aligned}
U(\boldsymbol{r}) & =-\left[\frac{1}{2}|\boldsymbol{\omega} \times \boldsymbol{r}|^{2}+\frac{1-\mu}{r_{1}}+\frac{\mu}{r_{2}}\right], \\
\boldsymbol{a}_{S} & =a_{0}(\boldsymbol{S} \cdot \boldsymbol{n})^{2} \boldsymbol{n}, \\
\boldsymbol{a}_{S E P} & =a_{S E P} \boldsymbol{m},
\end{aligned}
$$

where $\mu=0.1215$ is the mass ratio for the Earth-Moon system. The hybrid sail position vectors w.r.t. $m_{1}$ and $m_{2}$ respectively (see Figure 1 (a)) are $\boldsymbol{r}_{1}=[x+\mu, y, z]^{T}$ and $\boldsymbol{r}_{2}=[x-(1-\mu), y, z]^{T}, a_{0}$ is the magnitude of the solar radition pressure acceleration exerted on the hybrid sail and the unit vector $\boldsymbol{n}$ denotes the thrust direction, $\boldsymbol{a}_{S E P}$ is the acceleration from the SEP system and the unit vector $\boldsymbol{m}$ denotes the thrust direction. A constant displacement distance of $1750 \mathrm{~km}$ has been imposed, considering a characteristic acceleration of $a_{0}=0.10 \mathrm{~mm} / \mathrm{s}^{2}$ for the simulations. The sail is oriented such that it is always directed along the Sun-line $\boldsymbol{S}$, pitched at an angle $\gamma$ to provide a constant out-of-plane force. The unit normal to the hybrid sail surface $\boldsymbol{n}$ and the Sun-line direction are given by

$$
\begin{aligned}
\boldsymbol{n} & =\left[\begin{array}{lll}
\cos (\gamma) \cos \left(\omega_{\star} t\right) & -\cos (\gamma) \sin \left(\omega_{\star} t\right) & \sin (\gamma)
\end{array}\right]^{T}, \\
\boldsymbol{S} & =\left[\begin{array}{lll}
\cos \left(\omega_{\star} t\right) & -\sin \left(\omega_{\star} t\right) & 0
\end{array}\right]^{T},
\end{aligned}
$$

where $\omega_{\star}=0.923$ is the angular rate of the Sun-line in the corotating frame in a dimensionless synodic coordinate system.

\section{II.B. Linearized System}

We now want to investigate the dynamics of the hybrid sail in the neighborhood of the libration points. We denote the coordinates of the equilibrium point as $\boldsymbol{r}_{L}=\left(x_{L_{i}}, y_{L_{i}}, z_{L_{i}}\right)$ with $i=1, \cdots, 5$. Let a small displacement in $\boldsymbol{r}_{L}$ be $\delta \boldsymbol{r}$ such that $\boldsymbol{r} \rightarrow \boldsymbol{r}_{L}+\delta \boldsymbol{r}$. The equations for the hybrid sail can then be written as

$$
\frac{d^{2} \delta \boldsymbol{r}}{d t^{2}}+2 \boldsymbol{\omega} \times \frac{d \delta \boldsymbol{r}}{d t}+\nabla U\left(\boldsymbol{r}_{L}+\delta \boldsymbol{r}\right)=\boldsymbol{a}_{S}\left(\boldsymbol{r}_{L}+\delta \boldsymbol{r}\right)+\boldsymbol{a}_{S E P}\left(\boldsymbol{r}_{L}+\delta \boldsymbol{r}\right)
$$

and retaining only the first-order term in $\delta \boldsymbol{r}=[\delta x, \delta y, \delta y]^{T}$ in a Taylor-series expansion, the gradient of the potential and the acceleration can be expressed as

$$
\begin{aligned}
\nabla U\left(\boldsymbol{r}_{L}+\delta \boldsymbol{r}\right) & =\nabla U\left(\boldsymbol{r}_{L}\right)+\left.\frac{\partial \nabla U(\boldsymbol{r})}{\partial \boldsymbol{r}}\right|_{\boldsymbol{r}=r_{L}} \delta \boldsymbol{r}+O\left(\delta \boldsymbol{r}^{2}\right), \\
\boldsymbol{a}_{S}\left(\boldsymbol{r}_{L}+\delta \boldsymbol{r}\right) & =\boldsymbol{a}_{S}\left(\boldsymbol{r}_{L}\right)+\left.\frac{\partial \boldsymbol{a}_{S}(\boldsymbol{r})}{\partial \boldsymbol{r}}\right|_{\boldsymbol{r}=\boldsymbol{r}_{L}} \delta \boldsymbol{r}+O\left(\delta \boldsymbol{r}^{2}\right), \\
\boldsymbol{a}_{S E P}\left(\boldsymbol{r}_{L}+\delta \boldsymbol{r}\right) & =\boldsymbol{a}_{S E P}\left(\boldsymbol{r}_{L}\right)+\left.\frac{\partial \boldsymbol{a}_{S E P}(\boldsymbol{r})}{\partial \boldsymbol{r}}\right|_{\boldsymbol{r}=\boldsymbol{r}_{L}} \delta \boldsymbol{r}+O\left(\delta \boldsymbol{r}^{2}\right) .
\end{aligned}
$$

It is assumed that $\nabla U\left(\boldsymbol{r}_{L}\right)=0$, and the accelerations $\boldsymbol{a}_{S}$ and $\boldsymbol{a}_{S E P}$ are constant with respect to the small displacement $\delta \boldsymbol{r}$, so that

$$
\begin{gathered}
\left.\frac{\partial \boldsymbol{a}_{S}(\boldsymbol{r})}{\partial \boldsymbol{r}}\right|_{\boldsymbol{r}=r_{L}}=0, \\
\left.\frac{\partial \boldsymbol{a}_{S E P}(\boldsymbol{r})}{\partial \boldsymbol{r}}\right|_{r=r_{L}}=0 .
\end{gathered}
$$

The linear variational system associated with the libration points at $\boldsymbol{r}_{L}$ can be determined through a Taylor series expansion by substituting Eqs. (7), (8) and (9) into (6) so that

$$
\frac{d^{2} \delta \boldsymbol{r}}{d t^{2}}+2 \boldsymbol{\omega} \times \frac{d \delta \boldsymbol{r}}{d t}-K \delta \boldsymbol{r}=\boldsymbol{a}_{S}\left(\boldsymbol{r}_{L}\right)+\boldsymbol{a}_{S E P}\left(\boldsymbol{r}_{L}\right)
$$


where the matrix $K$ is defined as

$$
K=-\left[\left.\frac{\partial \nabla U(\boldsymbol{r})}{\partial \boldsymbol{r}}\right|_{r=r_{L}}\right]
$$

Using matrix notation the linearized equation about the libration point (Equation (12)) can be represented by the inhomogeneous linear system $\dot{\mathbf{X}}=A \mathbf{X}+\boldsymbol{b}(t)$, where the state vector $\mathbf{X}=(\delta \boldsymbol{r}, \delta \dot{\boldsymbol{r}})^{T}$, and for which $\boldsymbol{b}(t)$ (a $6 \times 1$ vector) is equal to the sum of control accelerations of the sail and the SEP.

The Jacobian matrix $A$ has the general form

$$
A=\left(\begin{array}{cc}
0_{3} & I_{3} \\
K & \Omega
\end{array}\right),
$$

where $I_{3}$ is a identity matrix, and

$$
\Omega=\left(\begin{array}{rrr}
0 & 2 & 0 \\
-2 & 0 & 0 \\
0 & 0 & 0
\end{array}\right)
$$

By making the transformation $\boldsymbol{r} \rightarrow \boldsymbol{r}_{L}+\delta \boldsymbol{r}$ and retaining only the first-order term in $\delta \boldsymbol{r}=(\xi, \eta, \zeta)^{T}$ in a Taylor-series expansion where $(\xi, \eta, \zeta)$ are axes attached to the libration point as shown in Figure 1 (a), the linearized nondimensional equations of motion relative to the collinear libration points can be written as

$$
\begin{aligned}
\ddot{\xi}-2 \dot{\eta}-U_{x x}^{o} \xi & =a_{\xi}+a_{S E P_{\xi}}, \\
\ddot{\eta}+2 \dot{\xi}-U_{y y}^{o} \eta & =a_{\eta}+a_{S E P_{\eta}}, \\
\ddot{\zeta}-U_{z z}^{o} \zeta & =a_{\zeta}+a_{S E P_{\zeta}}
\end{aligned}
$$

where $U_{x x}^{o}, U_{y y}^{o}$, and $U_{z z}^{o}$ are the partial derivatives of the gravitational potential evaluated at the collinear libration point, and the solar sail acceleration is defined in terms of three auxiliary variables $a_{\xi}, a_{\eta}$, and $a_{\zeta}$.

Again, the sail attitude is fixed such that the sail normal vector $\boldsymbol{n}$, which is the unit vector that is perpendicular to the sail surface, points always along the direction of the Sun line with the following constraint $\boldsymbol{S} \cdot \boldsymbol{n} \geq 0$. Its direction is described by the pitch angle $\gamma$ relative to the Sun-line, which represents the sail attitude.

Substituting Eqs. (4) and (5) into Eq. (2), the solar sail acceleration components are therefore given by

$$
\begin{aligned}
& a_{\xi}=a_{0} \cos \left(\omega_{\star} t\right) \cos ^{3}(\gamma) \\
& a_{\eta}=-a_{0} \sin \left(\omega_{\star} t\right) \cos ^{3}(\gamma) \\
& a_{\zeta}=a_{0} \cos ^{2}(\gamma) \sin (\gamma)
\end{aligned}
$$

where $a_{0}$ is the characteristic acceleration. The SEP acceleration components $\boldsymbol{a}_{S E P}$ are used for feedback control as described later.

By taking $\boldsymbol{a}_{S E P}=0$ (pure sail at linear order), Eqs. (16) - (18) have a simple periodic solution with a constant out-of-plane displacement of the form

$$
\begin{aligned}
\xi(t) & =\xi_{0} \cos \left(\omega_{\star} t\right), \\
\eta(t) & =\eta_{0} \sin \left(\omega_{\star} t\right), \\
\zeta(t) & =\zeta_{0} .
\end{aligned}
$$

By inserting equations (22) and (23) in the differential equations (16) and (17) with $\boldsymbol{a}_{S E P}=0$, we obtain the linear system in $\xi_{0}$ and $\eta_{0}$,

$$
\begin{gathered}
\left(U_{x x}^{o}-\omega_{\star}^{2}\right) \xi_{0}-2 \omega_{\star} \eta_{0}=a_{0} \cos ^{3}(\gamma), \\
-2 \omega_{\star} \xi_{0}+\left(U_{y y}^{o}-\omega_{\star}^{2}\right) \eta_{0}=-a_{0} \cos ^{3}(\gamma) .
\end{gathered}
$$

Then the amplitudes $\xi_{0}$ and $\eta_{0}$ are given by 


$$
\begin{aligned}
\xi_{0}=a_{0} \frac{\left(U_{y y}^{o}-\omega_{\star}^{2}-2 \omega_{\star}\right) \cos ^{3}(\gamma)}{\left(U_{x x}^{o}-\omega_{\star}^{2}\right)\left(U_{y y}^{o}-\omega_{\star}^{2}\right)-4 \omega_{\star}^{2}}, \\
\eta_{0}=a_{0} \frac{\left(-U_{x x}^{o}+\omega_{\star}^{2}+2 \omega_{\star}\right) \cos ^{3}(\gamma)}{\left(U_{x x}^{o}-\omega_{\star}^{2}\right)\left(U_{y y}^{o}-\omega_{\star}^{2}\right)-4 \omega_{\star}^{2}},
\end{aligned}
$$

and we have the equality

$$
\frac{\xi_{0}}{\eta_{0}}=\frac{\omega_{\star}^{2}+2 \omega_{\star}-U_{y y}^{o}}{-\omega_{\star}^{2}-2 \omega_{\star}+U_{x x}^{o}} .
$$

Then with condition (28), equations (22)-(24) will be used as a reference trajectory for the control analysis in the following sections. By applying a Laplace transform, the uncoupled out-of-plane $\zeta$-motion defined by the equation (18) can be expressed as (see Simo and $\mathrm{McInnes}^{29}$ for a detailed description)

$$
\zeta_{0}=a_{0} \cos ^{2}(\gamma) \sin (\gamma)\left|U_{z z}^{o}\right|^{-1} .
$$

Furthermore, the out-of-plane distance can be maximized by an optimal choice of the sail pitch angle determined by

$$
\begin{aligned}
\left.\frac{d}{d \gamma} \cos ^{2}(\gamma) \sin (\gamma)\right|_{\gamma=\gamma^{\star}} & =0 \\
\gamma^{\star} & =35.264^{\circ} .
\end{aligned}
$$

\section{Tracking by Feedback Linearization}

\section{III.A. Description}

Linearization by feedback is a well-known approach to control nonlinear systems. This method transforms a nonlinear state space model into a new coordinate system where the nonlinearities can be cancelled by feedback. It is a way of transforming system models into equivalent models of simpler form. For example, a change of variables $\boldsymbol{Z}=\Phi(\boldsymbol{X})$ is used to transform the state equation from the $\boldsymbol{X}$-coordinates to the $\boldsymbol{Z}$-coordinates, where the map $\Phi($.$) must be invertible, such that \boldsymbol{X}=\Phi^{-1}(\boldsymbol{Z})$ for $\boldsymbol{Z} \in \Phi(D)$ where $D$ is the domain of $\Phi$. Furthermore, the derivatives of $\boldsymbol{X}$ and $\boldsymbol{Z}$ should be continous and therefore the map $\Phi$ and its inverse $\Phi^{-1}($.$) are continously differentiable. Such a map is a diffeomorphism and can be viewed as$ a generalization of the coordinate transformation.

\section{III.B. Objectives}

Given the nonlinear system $\ddot{\boldsymbol{X}}=f(\boldsymbol{X}, \dot{\boldsymbol{X}})+\boldsymbol{u}$, the problem of feedback linearization consists of finding, if possible, a change of coordinates of the form $\boldsymbol{Z}=\Phi(\boldsymbol{X})$ and a static state feedback control $\boldsymbol{u}=(\boldsymbol{X}, \boldsymbol{\nu})$, such that the new control input $\boldsymbol{\nu}$ satisfy a linear time-invariant relation $\dot{\boldsymbol{Z}}=A \boldsymbol{Z}+B \boldsymbol{\nu}$ where the pair $(A, B)$ is controllable. This technique is completely different from a Jacobian linearization, on which linear control is based. From equation (1) the motion of the hybrid solar sail in the CRTBP is described by the scalar equations in the form

$$
\begin{aligned}
\ddot{\xi} & =2 \dot{\eta}+\left(x_{L_{i}}+\xi\right)-(1-\mu) \frac{\left(x_{L_{i}}+\xi\right)+\mu}{r_{1}^{3}}-\mu \frac{\left(x_{L_{i}}+\xi\right)-1+\mu}{r_{2}^{3}}+a_{\xi}+u_{\xi}, \\
\ddot{\eta} & =-2 \dot{\xi}+\eta-\left(\frac{1-\mu}{r_{1}^{3}}+\frac{\mu}{r_{2}^{3}}\right) \eta+a_{\eta}+u_{\eta}, \\
\ddot{\zeta} & =-\left(\frac{1-\mu}{r_{1}^{3}}+\frac{\mu}{r_{2}^{3}}\right) \zeta+a_{\zeta}+u_{\zeta},
\end{aligned}
$$


where the vector

$$
\boldsymbol{u}(t)=\left[\begin{array}{lll}
u_{\xi} & u_{\eta} & u_{\zeta}
\end{array}\right]^{T}
$$

is the applied control acceleration due to the SEP thruster, such that $\boldsymbol{u}(t) \triangleq \boldsymbol{a}_{S E P}$.

To develop a feedback linearization scheme, the motion of the hybrid solar sail moving in the CRTBP is separated into linear and nonlinear components, such that

$$
\begin{aligned}
\ddot{\xi} & =f_{\text {Non-Linear }}^{\xi}+f_{\text {Linear }}^{\xi}+a_{\xi}+u_{\xi}, \\
\ddot{\eta} & =f_{\text {Non-Linear }}^{\eta}+f_{\text {Linear }}^{\eta}+a_{\eta}+u_{\eta}, \\
\ddot{\zeta} & =f_{\text {Non-Linear }}^{\zeta}+f_{\text {Linear }}^{\zeta}+a_{\zeta}+u_{\zeta},
\end{aligned}
$$

where the $f$ functions are defined as the linear and the nonlinear terms in the equations (32), (33) and (34)

$$
\begin{aligned}
f_{\text {Non-Linear }}^{\xi} & =-(1-\mu) \frac{\left(x_{L_{i}}+\xi\right)+\mu}{r_{1}^{3}}-\mu \frac{\left(x_{L_{i}}+\xi\right)-1+\mu}{r_{2}^{3}}, \\
f_{\text {Linear }}^{\xi} & =2 \dot{\eta}+\left(x_{L_{i}}+\xi\right), \\
f_{\text {Non-Linear }}^{\eta} & =-\left(\frac{1-\mu}{r_{1}^{3}}+\frac{\mu}{r_{2}^{3}}\right) \eta, \\
f_{\text {Linear }}^{\eta} & =-2 \dot{\xi}+\eta \\
f_{\text {Non-Linear }}^{\zeta} & =-\left(\frac{1-\mu}{r_{1}^{3}}+\frac{\mu}{r_{2}^{3}}\right) \zeta, \\
f_{\text {Linear }}^{\zeta} & =0,
\end{aligned}
$$

with $r_{1}=\sqrt{\left(\left(x_{L_{i}}+\xi\right)+\mu\right)^{2}+\eta^{2}+\zeta^{2}} \quad$ and $\quad r_{2}=\sqrt{\left(\left(x_{L_{i}}+\xi\right)-1+\mu\right)^{2}+\eta^{2}+\zeta^{2}}$.

The solar sail acceleration components are given in equations (19), (20) and (21). We then select the SEP control $\boldsymbol{u}(t)$ such that

$$
\boldsymbol{u}(t)=\left[\begin{array}{l}
u_{\xi} \\
u_{\eta} \\
u_{\zeta}
\end{array}\right]=\boldsymbol{U}(t)+\tilde{\boldsymbol{u}}(t)
$$

where

$$
\boldsymbol{U}(t)=-\left[\begin{array}{rr}
\left(x_{L_{2}}+\xi\right)-(1-\mu) \frac{\left(x_{L_{2}}+\xi\right)+\mu}{r_{1}^{3}} & -\mu \frac{\left(x_{L_{2}}+\xi\right)-1+\mu}{r_{2}^{3}}-U_{x x}^{o} \xi \\
& -\left(\frac{1-\mu}{r_{1}^{3}}+\frac{\mu}{r_{2}^{3}}\right) \eta-U_{y y}^{o} \eta \\
& -\left(\frac{1-\mu}{r_{1}^{3}}+\frac{\mu}{r_{2}^{3}}\right) \zeta-U_{z z}^{o} \zeta
\end{array}\right]
$$

is the canceling term and $\tilde{\boldsymbol{u}}(t)$ the stabilizing term.

The equations (32), (33) and (34) then become

$$
\begin{aligned}
\ddot{\xi} & =2 \dot{\eta}+U_{x x}^{o} \xi+a_{0} \cos \left(\omega_{\star} t\right) \cos ^{3}(\gamma)+\tilde{u}_{\xi}, \\
\ddot{\eta} & =-2 \dot{\xi}+U_{y y}^{o} \eta-a_{0} \sin \left(\omega_{\star} t\right) \cos ^{3}(\gamma)+\tilde{u}_{\eta}, \\
\ddot{\zeta} & =U_{z z}^{o} \zeta+a_{0} \cos ^{2}(\gamma) \sin (\gamma)+\tilde{u}_{\zeta} .
\end{aligned}
$$

By removing the nonlinear dynamics from the system, the control acceleration vector $\tilde{\boldsymbol{u}}(t)$ is determined such that the desired response characteristics of the linear time-invariant dynamics are produced and so Eq. (42) - (44) are identical to the linear system defined by Eq. (16) - (18). In particular, it can be ensured that the displacement distance of the periodic orbit is constant, which provides key advantages for lunar polar telecommunications. 


\section{Tracking a Reference Trajectory}

\section{IV.A. Linear Feedback Control}

Let us consider nonlinear system described by

$$
\ddot{\boldsymbol{x}}=f(\boldsymbol{x}, \dot{\boldsymbol{x}})+\boldsymbol{u},
$$

where $\boldsymbol{x} \in \mathbb{R}^{3}$ is the position. Let $\boldsymbol{e}(t)=\boldsymbol{x}(t)-\boldsymbol{x}_{r e f}(t)$ denote the position error relative to some reference solution, where the reference trajectory

$$
\boldsymbol{x}_{\text {ref }}(t)=\left[\begin{array}{lll}
\xi_{\text {ref }} & \eta_{\text {ref }} & \zeta_{\text {ref }}
\end{array}\right]^{T}
$$

is given by the analytical solution

$$
\begin{aligned}
\xi_{\text {ref }}(t) & =\xi_{0} \cos \left(\omega_{\star} t\right), \\
\eta_{\text {ref }}(t) & =\eta_{0} \sin \left(\omega_{\star} t\right), \\
\zeta_{\text {ref }}(t) & =\zeta_{0} .
\end{aligned}
$$

We then differentiate $\boldsymbol{e}(t)$ until the control appears so that

$$
\begin{aligned}
\boldsymbol{e}(t) & =\boldsymbol{x}(t)-\boldsymbol{x}_{r e f}(t), \\
\dot{\boldsymbol{e}}(t) & =\dot{\boldsymbol{x}}(t)-\dot{\boldsymbol{x}}_{r e f}(t), \\
\ddot{\boldsymbol{e}}(t) & =\ddot{\boldsymbol{x}}(t)-\ddot{\boldsymbol{x}}_{r e f}(t), \\
& =f(\boldsymbol{x}, \dot{\boldsymbol{x}})+\boldsymbol{u}-\ddot{\boldsymbol{x}}_{r e f}(t), \\
& =-\lambda_{1} \dot{\boldsymbol{e}}-\lambda_{2} \boldsymbol{e},
\end{aligned}
$$

and so, we have

$$
\boldsymbol{u}(t)=-f(\boldsymbol{x}, \dot{\boldsymbol{x}})+\ddot{\boldsymbol{x}}_{r e f}(t)-\lambda_{1} \dot{\boldsymbol{e}}-\lambda_{2} \boldsymbol{e},
$$

where

$$
f=\left[\begin{array}{l}
f_{\text {Non-Linear }}^{\xi} \\
f_{N o n-L i n e a r}^{\eta} \\
f_{\text {Non-Linear }}^{\zeta}
\end{array}\right]
$$

and $-\lambda_{1} \dot{\boldsymbol{e}}-\lambda_{2} \boldsymbol{e}$ is the stabilizing term.

\section{IV.B. Trajectory Tracking}

Consider the system given by equation (45), where our objective is to make the output $\boldsymbol{x} \in \mathbb{R}^{3}$ track a desired trajectory given by the reference trajectory $\boldsymbol{x}_{r e f} \in \mathbb{R}^{3}$ while keeping the position bounded. Therefore, we want to find a control law for the input $\tilde{\boldsymbol{u}} \in \mathbb{R}^{3}$ such that, starting from any initial position in a domain $D \subset \mathbb{R}^{3}$, the tracking error $\boldsymbol{e}(t)=\boldsymbol{x}(t)-\boldsymbol{x}_{r e f}(t)$ goes to zero. Hence, asymptotic tracking will be achieved if we design a state feedback control law to ensure that $\boldsymbol{e}(t)$ is bounded and converges to zero as $t$ tends to infinity. Thus, the control law

$$
\tilde{\boldsymbol{u}}=-\lambda_{1} \dot{\boldsymbol{e}}-\lambda_{2} \boldsymbol{e}
$$

yields the tracking error equation

$$
\ddot{e}+\lambda_{1} \dot{e}+\lambda_{2} e=0,
$$

where $\lambda_{1}$ and $\lambda_{2}$ are chosen positive constants. 


\section{Evaluation of Hybrid Sail Performance}

\section{V.A. Evaluation}

In this section we investigate the performance of the hybrid sail system, constituted by a solar sail combined with solar electric propulsion. The simulation was performed around the collinear libration point $L_{2}$ for a period of one month. Thus, the control acceleration effort $\boldsymbol{U}(t)$ required to track the reference orbit while rejecting the nonlinearities varies up to $0.014 \mathrm{~mm} / \mathrm{s}^{2}$ about the $L_{2}$ point. The control accelerations are continous smooth signals. The acceleration derived from the solar sail (denoted by $a_{\xi}, a_{\eta}, a_{\zeta}$ ) is plotted in terms of components for one-month orbits in Figure 2 (a) about $L_{2}$, and the SEP acceleration components appears in Figure 2 (b) about $L_{2}$. The control acceleration effort derived from the thruster (denoted by $U_{\xi}$, $\left.U_{\eta}, U_{\zeta}\right)$ is order of $10^{-3}-10^{-4} \mathrm{~mm} / \mathrm{s}^{2}$, while the acceleration derived from the solar sail is approximately $10^{-2} \mathrm{~mm} / \mathrm{s}^{2}$. The small control acceleration from the SEP thruster is then applied to ensure that the displacement of the periodic orbit is constant. The solar sail provides a constant out-of-plane force. Figure 3 (critically damped motion) illustrates the position error components, denoted by $e_{\xi}, e_{\eta}, e_{\zeta}$ under the nonlinear control and the SEP thruster around $L_{2}$. These Figures show that the motion is bounded and periodic. This observation implies that the augmented thrust acceleration ensures a constant displacement orbit.
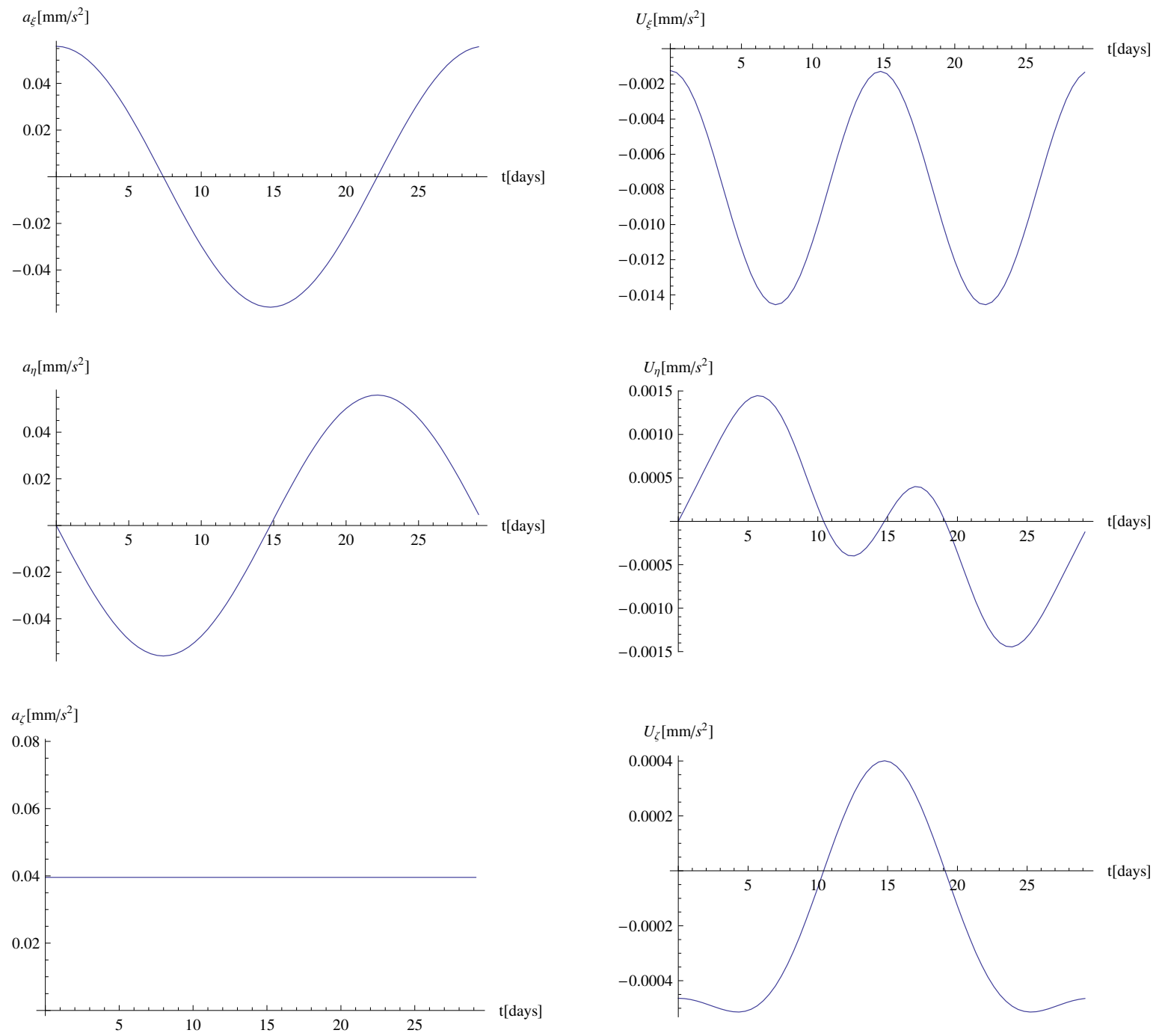

(a)

$(b)$

Figure 2. (a) Acceleration derived from the solar sail about the $L_{2}$ point; (b) Acceleration derived from the SEP thruster about the $L_{2}$ point.

The parameters of the reference trajectory used for the simulations are summarized in Table 1. 


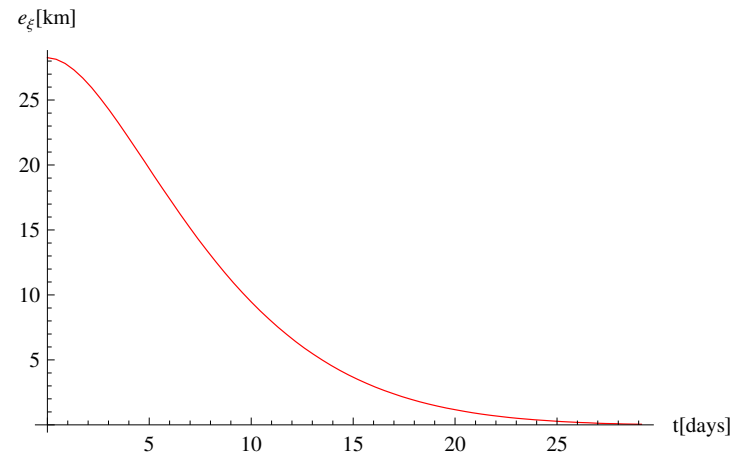

(a)

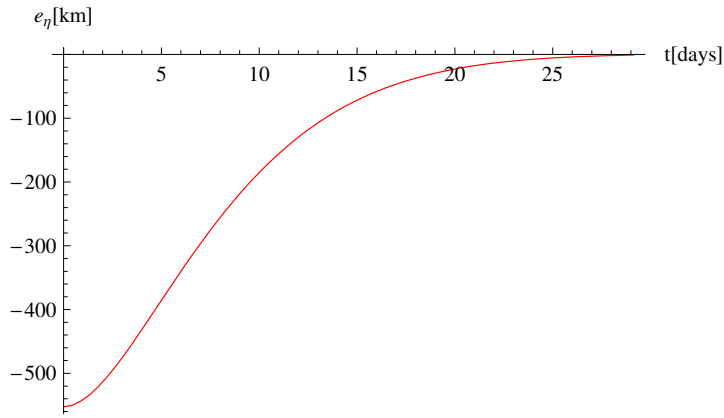

(b)

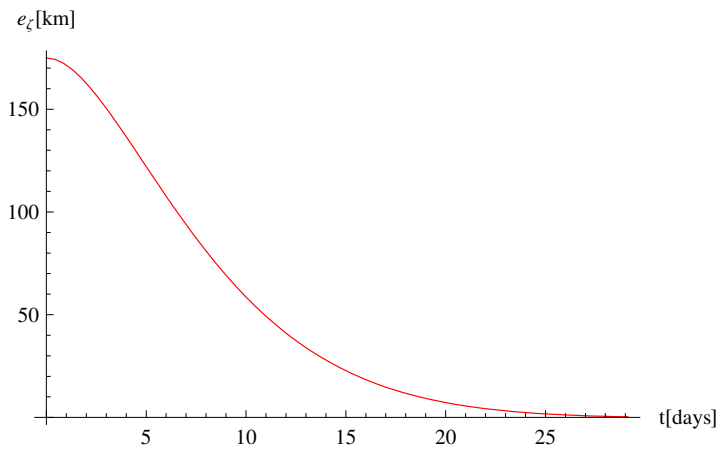

(c)

Figure 3. Position error components about the $L_{2}$ point with $e(0)=(28.26,-552.52,175)^{T} k m$ (Critically damped motion): (a), (b) and (c).

Table 1. Parameters of Reference Trajectory.

\begin{tabular}{llccr}
\hline & $\xi_{0}[\mathrm{~km}]$ & $\eta_{0}[\mathrm{~km}]$ & $\zeta_{0}[\mathrm{~km}]$ & $a_{0}\left[\mathrm{mms}^{-2}\right]$ \\
\hline & & & & \\
$L_{2}$ & 282.613 & -5525.23 & 1750 & 0.10 \\
\hline
\end{tabular}




\section{V.B. Propellant Usage}

Propellant usage for the SEP thruster is proportional to the total $\Delta V$, which is the integration over time of the magnitude of the control acceleration acceleration produced by using the SEP thruster so that

$$
\Delta V=\int_{0}^{2 \pi / \omega_{\star}}|\boldsymbol{u}| d t
$$

The total $\Delta V_{\text {Total }}$ over a 5 year mission is given by

$$
\Delta V_{\text {Total }}=\Delta \text { Vper orbit } \times \text { no, }
$$

where no is the total number of orbits. Once the total $\Delta V$ is computed, the propellant usage can be found using the rocket equation.

Let us define the mass $m$ of the system at a time $t$, as a function of the initial mass $m_{i}, \Delta V$ and the effective exhaust velocity $v_{e}=I_{s p} \cdot g$,

$$
m=m_{i} e^{-\Delta V / g \cdot I_{s p}}
$$

The mass of propellant is then the difference between the initial and the final masses

$$
m_{\text {prop }}=m_{i}-m=m_{i}\left(1-e^{-\Delta V_{\text {Total }} / g \cdot I_{s p}}\right),
$$

where $I_{s p}$ is the specific impulse $(\approx 3000 \mathrm{sec}$ for an electric thruster).

Assume a specific impulse of $I_{s p}=3000 \mathrm{sec}$ and an initial mass of $m_{i}=500 \mathrm{~kg}$, we have the average $\Delta V$ per orbit of approximately $23 \mathrm{~m} / \mathrm{s}$ for motion about $L_{2}$. Then, the total $\Delta V$ per orbit over 5 years is 1536 $\mathrm{m} / \mathrm{s}$. The consumed propellant mass is then $m_{\text {prop }}=25 \mathrm{~kg}$. The parameters are summarized in Table 2 .

Table 2. Summary of Parameters.

\begin{tabular}{ccr}
\hline Parameter & Description & Value \\
\hline$m_{i}(k g)$ & Initial Mass & 500 \\
$I_{s p}(\mathrm{sec})$ & Specific Impulse & 3000 \\
$\Delta V_{\text {Total }}(\mathrm{m} / \mathrm{s})$ & Total $\Delta V$ over 5 years & 1536 \\
$m_{\text {prop }}(\mathrm{kg})$ & Propellant Mass Consummed & 25 \\
\hline
\end{tabular}

\section{Conclusions}

A hybrid concept for displaced periodic orbits in the Earth-Moon system has been developed. A feedback linearization was used to perform stabilization and trajectory tracking for the nonlinear system. The idea of this control is to transform a given nonlinear system into a linear system by use of a nonlinear coordinate transformation and nonlinear feedback. The augmented thrust acceleration is than applied to ensure a constant displacement periodic orbit, which provides key advantages for lunar polar telecommunications. A stabilizing approach is then introduced to increase the damping in the system and to allow a higher gain in the controller. Theoretical and simulation results show good performance, with modest propellant mass requirements.

\section{Acknowledgments}

This work was funded by the European MCRTN (Marie Curie Research Training Network) AstroNet, Contract Grant No. MRTN-CT-2006-035151. 


\section{References}

226.

${ }^{1}$ McInnes, C. R., Solar sailing: technology, dynamics and mission applications, Springer Praxis, London, 1999, pp. 214-

${ }^{2}$ Waters, T. and McInnes, C., "Periodic Orbits Above the Ecliptic in the Solar-Sail Restricted Three-Body Problem," J. of Guidance, Control, and Dynamics, Vol. 30, No. 3, 2007, pp. 687-693.

${ }^{3}$ Simo, J. and McInnes, C. R., "Solar sail trajectories at the Earth-Moon Lagrange points," In 59th International Astronautical Congress, Glasgow, Scotland, 29 Sep - 03 Oct 2008. Paper IAC-08.C1.3.13.

${ }^{4}$ McInnes, C., "Solar sail Trajectories at the Lunar L 2 Lagrange Point," J. of Spacecraft and Rocket, Vol. 30, No. 6, 1993, pp. $782-784$.

${ }^{5}$ Ozimek, M., Grebow, D., and Howell, K., "Solar Sails and Lunar South Pole Coverage," In AIAA/AAS Astrodynamics Specialist Conference and Exhibit, Honolulu, Hawaii,August 2008. Paper AIAA-2008-7080.

${ }^{6}$ Baoyin, H. and McInnes, C., "Solar sail halo orbits at the Sun-Earth artificial L 1 point," Celestial Mechanics and Dynamical Astronomy, Vol. 94, No. 2, 2006, pp. 155-171.

${ }^{7}$ Baoyin, H. and McInnes, C., "Solar sail equilibria in the elliptical restricted three-body problem," Journal of Guidance, Control and Dynamics, Vol. 29, No. 3, 2006, pp. 538-543.

${ }^{8}$ Baoyin, H. and McInnes, C., "Solar sail orbits at artificial Sun-Earth Lagrange points," Journal of Guidance, Control and Dynamics, Vol. 28, No. 6, 2005, pp. 1328-1331.

${ }^{9}$ McInnes, C. R., "Artificial Lagrange points for a non-perfect solar sail," Journal of Guidance, Control and Dynamics, Vol. 22, No. 1, 1999, pp. 185-187.

${ }^{10}$ McInnes, C., McDonald, A., Simmons, J., and McDonald, E., "Solar sail parking in restricted three-body systems," Journal of Guidance, Control and Dynamics, Vol. 17, No. 2, 1994, pp. 399-406.

${ }^{11}$ Leipold, M. and Götz, M., "Hybrid Photonic/Electric Propulsion," Kayser-Threde, TR SOL4- TR-KTH-0001, Munich, Jan. 2002, ESA Contract No. 15334/01/NL/PA.

${ }^{12}$ Mengali, G. and Quarta, A. A., "Trajectory Design with Hybrid Low-Thrust Propulsion system," Journal of Guidance, Control, and Dynamics, Vol. 30, No. 2, March-April 2007, pp. 419-426.

${ }^{13}$ Dachwald, B., "Optimization of Very-Low-Thrust Trajectories Using Evolutionary Neurocontrol," Acta Astronautica, Vol. 57, 2005, pp. 175-185.

${ }^{14}$ Baig, S. and McInnes, C., "Artificial Three-Body Equilibria for Hybrid Low-Thrust Propulsion," Journal of Guidance, Control, and Dynamics, Vol. 31, No. 6, November-December 2008, pp. 1644-1655.

${ }^{15}$ Szebehely, V., Theory of Orbits: the restricted problem of three bodies, Academic Press, New York and London, 1967, pp. 497-525.

${ }^{16}$ Farquhar, R., "The Control and Use of Libration-Point Satellites," Ph.D. Dissertation, Stanford University, 1968.

${ }^{17}$ Roy, A. E., Orbital Motion, Institute of Physics Publishing, Bristol and Philadelphia, 2005, pp. 118-130.

${ }^{18}$ Vonbun, F., "'A Humminbird for the $\mathrm{L}_{2}$ Lunar Libration Point"," Nasa TN-D-4468, April 1968.

${ }^{19}$ Thurman, R. and Worfolk, P., "The geometry of halo orbits in the circular restricted three-body problem," Technical report GCG95, Geometry Center, University of Minnesota, 1996.

${ }^{20}$ Gómez, G., Llibre, J., Martínez, R., and Simó, C., Dynamics and Mission Design Near Libration Points, Vol. I, II, World Scientific Publishing Co.Pte.Ltd, Singapore.New Jersey.London.Hong Kong, 2001.

${ }^{21}$ Gómez, G., Jorba., A., J.Masdemont, and Simó, C., Dynamics and Mission Design Near Libration Points, Vol. III, IV, World Scientific Publishing Co.Pte.Ltd, Singapore.New Jersey.London.Hong Kong, 2001.

${ }^{22}$ Breakwell, J. and Brown, J., "The 'halo' family of 3-dimensional periodic orbits in the Earth-Moon restricted 3-body problem," Celestial Mechanics, Vol. 20, 1979, pp. 389-404.

${ }^{23}$ Richardson, D. L., "Halo orbit formulation for the ISEE-3 mission," J. Guidance and Control, Vol. 3, No. 6, 1980, pp. 543-548.

${ }^{24}$ Howell, K., "Three-dimensional, periodic, 'halo' orbits," Celestial Mechanics, Vol. 32, 1984, pp. 53-71.

${ }^{25}$ Howell, K. and Marchand, B., "'Natural and Non-Natural Spacecraft Formations Near $\mathrm{L}_{1}$ and $\mathrm{L}_{2}$ Libration Points in the Sun-Earth/Moon Ephemerics System"," Dynamical Systems: An International Journal, Vol. 20, No. 1, March 2005, pp. 149-173.

${ }^{26}$ Farquhar, R., "The utilization of Halo orbits in advanced lunar operations," Nasa technical Note D-6365, 1971.

${ }^{27}$ Farquhar, R. and Kamel, A., "Quasi-periodic orbits about the translunar libration point," Celestial Mechanics, Vol. 7, 1973, pp. 458-473.

${ }^{28}$ Slotine, J.-J. E. and Li, W., Applied Nonlinear Control, Prentice Hall, Englewood Cliffs, New Jersey 07632, 1991, pp. 208-212.

${ }^{29}$ Simo, J. and McInnes, C. R., "Solar sail orbits at the Earth-Moon Libration points," Communications in Nonlinear Science and Numerical Simulation, March 2009 [in press]. doi: 10.1016/j.cnsns.2009.03.032. 